\title{
Apuntes para la historia de la Unidad Académica de Medicina de la Universidad Autónoma de Nayarit ${ }^{1}$.
}

\section{Notes for the history of the Academic Unit of Medicine of the Autonomous University of Nayarit}

DOI: $10.46932 / \mathrm{sfjdv} 2 \mathrm{n} 4-052$

Received in: March 1st, 2021

Accepted in: May 30th, 2021

\author{
Bernabe Rios Nava \\ PHD in education \\ Institution: universidad autonoma de nayarit \\ Address: av. De la cultura s/n. Col. Centro. Cp 63000. Tepic, nayarit, mexico. \\ E-mail: brios402017@gmail.com
}

"[...] dentro de la universidad no habia un edificio disponible [...] no recuerdo detalles[...] del edificio, [...] con el apoyo de estas gentes y del rector [...] llegó un buen día que nos dijeron, (que) esa iba ser nuestra escuela y por ahí circula la famosa fotografía del primer día, en que llegamos a ese edificio "tomado" por toda la "bola",

en la calle Lerdo antes de entrar al edificio, ese fue nuestro primer día que pisamos[...]

la famosa "Casa Fenelón".

\section{RESUMEN}

Los pasados 20 al 23 de Mayo de 2013 evaluadores del Consejo Mexicano para la Acreditación de la Educación Médica (COMAEM), realizaron su proceso de evaluación para la re-acreditación del programa de estudios de la Unidad Académica de Medicina (UAM) de la Universidad Autónoma de Nayarit (UAN). El 8 de noviembre de 2013 en ceremonia oficial, le fue entregada al rector la placa de reconocimiento. Este hecho coloca a la institución como la más importante en la formación médica de pregrado en el estado. El presente trabajo vuelve al pasado y recupera algunas de sus etapas históricas más importantes.

Palabras clave: Educación médica, programas de estudio, programas educativos, medicina, historia de la educación.

\begin{abstract}
The past 20 al May 23, 2013 evaluators of the Mexican Council for Accreditation of Medical Education (COMAEM) conducted their evaluation process for re-accreditation of the curriculum of the Academic Unit of Medicine (UAM), Autonomus University of Nayarit (UAN). On November 8, 2013 in an official ceremony, the rector was given recognition plaque. This fact puts the institution as the most important in undergraduate medical education in the state. This paper returns to the past and recovers some of its most important historical stages.
\end{abstract}

Key words: Medical education, curriculum, educational programs, medicine, history of education.

\footnotetext{
${ }^{1}$ El presente trabajo retoma partes sustanciales del capítulo IV de la tesis de doctorado presentada en la Universidad Autónoma de Aguascalientes, en el año de 2006.
} 


\section{INTRODUCCIÓN}

El lunes 8 de julio de 1974, en la sesión celebrada por el H. Consejo General Universitario se dio lectura al proyecto para la creación de la Escuela Superior de Medicina² (hoy Unidad Académica de Medicina) (UAM) de la Universidad de Nayarit (UNINAY) hoy Universidad Autónoma de Nayarit (UAN), el cual fue aprobado por unanimidad y se designó al mes de marzo del año de 1975, como la fecha para el inicio de sus actividades. La cual coincide con el año de mayor apertura de escuelas de medicina en nuestro país $(7)^{3}$, pasando de 39 en 1974 a 46. Este hecho representó un momento trascendental para la vida académica universitaria de la región y del país, por dos motivos: la universidad nayarita abría un espacio educativo a los jóvenes recién egresados del nivel medio superior, contribuyendo a contener el flujo migratorio ${ }^{4}$ de estos, hacia otras universidades del occidente y centro del país; y segundo, se incrementaba a nivel nacional, el número de escuelas y facultades de medicina con programas académicos de corte modular. La (UAM) acoge el "Programa de Medicina General Integral A-36 (PMGI-A-36) 5", que hacía algunos meses - década de los setentas- había ofertado como segunda opción la Facultad de Medicina de la UNAM. El surgimiento de la (UAM), se da en un contexto nacional caracterizado: por la expansión de la educación superior mexicana; de gran efervescencia del movimiento médico educativo renovador, y dentro de un gran proceso de transformación universitaria nayarita el "Proyecto de Nueva Universidad", en el cual destacaban: la creación del Plan Experimental de Economía, el programa de “Unidades de Producción”, la elaboración del Estatuto Jurídico, y la conformación de las áreas: de Ingeniería, con sus ramas pesquera, agrícola, química industrial y veterinaria-zootecnia; Ciencias de la Salud, con medicina y enfermería; y Ciencias Sociales con economía, derecho, comercio, ciencias de la educación y turismo próximamente. Estos hechos representaron algunos frutos de las tareas de planeación universitaria, que en su momento fueron encomendadas al Instituto de Investigaciones Económicas. La apertura de la (UAM) se considera un proyecto educativo con profundas raíces colectivas; una obra al servicio de la comunidad, que contó con numerosas manos y una multitud de ideas de una generación de estudiantes egresados del bachillerato de Ciencias de la Salud de la Preparatoria \# 1 de Tepic..."Las generaciones son los

\footnotetext{
${ }^{2}$ En adelante (UAM)

${ }^{3}$ Ese año se abre sus puertas; las escuelas de medicina de la Universidad Autónoma de Chiapas, Universidad Juárez de Durango, ENEP-I, Universidad Anáhuac, Universidad Autónoma del Estado de Morelos, y Universidad de Montemorelos. (Fernández ,1996).

${ }^{4}$ La presencia de la (UAN) y de la (UAM) contribuyeron en parte a frenar el fenómeno social de la emigración de estudiantes egresados de educación media superior -a otras universidades del centro del país-, que durante mucho tiempo vivieron las familias nayaritas. En una investigación del año de 1970, se concluía que por cada 100 estudiantes de medicina del país, 48 se localizaban en el D.F., hacia 1983 sólo eran 22 alumnos. Aún con la desconcentración estudiantil, el D.F. continuaba siendo la entidad con mayor número de estudiantes de medicina del país, captando más de la $5^{\mathrm{a}}$ parte de los estudiantes del país y más de la mitad estudian en la UNAM. (ANUIES, 1984).
}

${ }^{5}$ En adelante (A-36) 
actores de la historia, su relevo presenta la renovación de las claves y el eclipse de las preocupaciones de una época[...]”. (Barrón, 1997)

\section{DISEÑO METODOLÓGICO}

La reconstrucción de las etapas históricas fue posible a partir de los testimonios de egresados, docentes y funcionarios del gobierno estatal y universitarios. Se utilizó la entrevista a profundidad, particularmente la que proporciona una gama de escenarios, situaciones o personas (Taylor y Bogdan,1987). Su empleo se hizo con diversos propósitos: primero, como un medio idóneo de acercarse al conocimiento de los fenómenos sociales (García,1986), con el fin de acceder a datos e informaciones que traspasasen las líneas de lo habitual o lo conocido, y que nuestros entrevistados resignificaran, experiencias a partir del modo cómo han conformado su esquema referencial (Díaz,1995) y han encontrado un significado profundo de nuestras vidas (Atkinson,1998). Guardamos y cubrimos con rigurosidad el protocolo que diversos autores (Díaz,1995; Taylor y Bogdan,1985; García,1986; Padua,2002), señalan como indispensables: establecer un acuerdo mutuo, en el sentido de que las preguntas fueran solo una invitación a expresar con diferentes intensidades, puntos de vista, relatos, experiencias, apreciaciones y valoraciones sobre el tema de interés, de manera abierta y sin cortapisas. Además se fueron construyendo las condiciones y ambientes idóneos. La entrevista se acompañó de un "guion", una especie de lista de áreas generales o temas clave, la cual cubrió dos aspectos; el primero, que cada entrevistado tuviera conocimiento del número y tipo de preguntas a realizársele y segundo, que todos respondieran las mismas preguntas, lo que nos dio la libertad de decidir cómo y cuándo enunciarlas.

\subsection{EL ORIGEN}

Las primeras noticias sobre la apertura de la (UAM) aparecen en los periódicos de la época en el año de 1974, particularmente en la conferencia ofrecida por el entonces rector Ricardo Vidal Manzo, cuando explicaba los diversos proyectos que se realizarían al interior de algunas escuelas de la universidad: en la de Agricultura se crearían viveros y granjas de floricultura; en Ingeniería Pesquera, su planta de harina de pescado; en Medicina Veterinaria y Zootecnia, sus viveros de animales silvestres, plantas porcícolas; talleres en las escuelas preparatorias; la alberca universitaria y la creación de la escuela de medicina. (Zamudio, 1974)

Un hecho importante fue la creación del Instituto de Investigaciones Económicas (IIE) ${ }^{6}$ en

\footnotetext{
${ }^{6} \mathrm{El}$ instituto fue posible gracias a la petición de los alumnos y a un fondo aportado por la SEP, CONACYT y la UNINAY.
} (Salinas, 1977). 
marzo de 1974, dependiente de la Escuela de Economía; un centro de excelencia en principio conformado por dos áreas importantes: la economía de la educación, a fin de orientar la formación de cuadros de investigadores, el apoyo a las tareas de planeación universitarias y las investigaciones sobre desarrollo, a partir del conocimiento de las áreas potenciales del estado (Salinas,1977). La presencia del instituto marcó los destinos académicos de varias escuelas de la universidad, entre ellas la de Medicina; ayudó a forjar el recurso económico para el Proyecto de Nueva Universidad, a través del Programa de las "Unidades de Producción"

“[...] nos pidieron como primera tarea el planear la creación de la escuela de Medicina. Que era una demanda muy sentida de la población y de la comunidad universitaria desde hacía muchos años [...] lo que se hizo fue convocar a todos los interesados [...] del alumnado y de la planta académica para participar en una investigación que permitiera diseñar la escuela de Medicina que necesitaba el estado de Nayarit [...]”.' (Entrevista, Manuel Ulloa Herrero, 1998)

En la sesión del H. Consejo General Universitario del lunes 8 de julio de 1974, se da lectura al proyecto de creación de la Escuela de Medicina (el doctor David Trejo González presenta: la exposición de motivos (la necesidad de la formación de un profesional de la salud); los objetivos que se plantea la Escuela de Medicina; su estructura; el plan de estudios; personal académico que inicia; tipos de financiamientos de que dispone y conclusiones. El cual es aprobado por unanimidad, y se estipula el mes de marzo de 1975 para el inicio de sus actividades. (UAN, 1974)

Meses después (8 de julio 1974) en sesión extraordinaria del (CGU), se delinean los requisitos para la admisión al primer curso propedéutico: se admitirán un total de 200 estudiantes, considerando primeramente los egresados del bachillerato especializado de Ciencias de la Salud de la universidad; seguidos de los egresados de otros bachilleratos o planes de estudio; el curso iniciaría el 9 de septiembre concluyendo el 21 de diciembre, debiéndose pagar una cuota de \$200. De éste total se aceptaran a 144 estudiantes que conformarán los cuatro grupos de 36 estudiantes con los que iniciará la (UAM). (Diario del Pacífico, 1974)

Al paso de los meses, fueron detallándose otras de sus características, por ejemplo a inicios de 1975:

"[...]el plan de estudios de la Escuela de Medicina[...] está adecuado para la aplicación de los sistemas más actuales de enseñanza, de la técnica pedagógica más avanzada [...] establece formar médicos cirujanos generales, capacitados para atender prevención de la salud, diagnóstico, tratamiento y rehabilitación de la patrología más frecuente[...] co contará con cuatro maestros de tiempo completo[...] ]además con[...] catedráticos especialistas en las diferentes ramas [...] su preparación (no) va a ser memorista como la escuela tradicional[...] el de aquí no va a ser un profesionista que como sabio esté encerrado[...] estará vinculado a la comunidad para que con la práctica se forme un médico integral". (Sandoval, 1975) Se conforma la comisión pro-construcción de la escuela de medicina 
Una inquietud que reinaba entre el alumnado de ciencias de la salud de la preparatoria No. 1, era la incertidumbre de ¿dónde continuar estudiando? al término de sus estudios. Para esos momentos la (UAN), contaba con una oferta de carreras a nivel licenciatura muy limitada. En el área de ciencias de la salud, las opciones eran aún más restringidas; se contaba con la Escuela de Enfermería, creada en la década del cuarenta y la Escuela de Odontología, que había iniciado sus labores el 2 de septiembre de 1969.

Para quienes estas opciones no cubrían sus expectativas, tendrían que emigrar del estado, ya sea a la ciudad de Guadalajara o a la ciudad de México, y entrar a concurso en los procesos de selección de las facultades de medicina ${ }^{7}$. Esta situación curiosamente fue el motor que empezó a agrupar a diversos bachilleres, en torno de una idea que en su momento, se antojaba un tanto utópica: la creación de una nueva escuela de medicina. Sin embargo de fondo se tenía una espléndida justificación, su apertura podría evitar que los jóvenes con limitados recursos económicos -que eran la mayoríatuvieran que emigrar, optar por las opciones que ofrecía la universidad o dedicarse a otra actividad.

"[...] de hecho no teníamos otra salida, (si) queríamos estudiar medicina, teníamos que hacerlo aquí, el hecho de irnos fuera, pues estaba difícil por la cuestión económica "EE1

Como en pocas ocasiones, la creación de una escuela de medicina puede atribuirse a la idea de un grupo de alumnos preparatorianos, que junto a algunos docentes, empezaron a dar forma y acción a esta utopía posible. De este modo la (UAM), rompe con el estilo de las aperturas de escuelas superiores, que algunas mediante; decretos, acuerdos cupulares, dan cuenta de su existencia ${ }^{8}$. Siendo una idea de 30 estudiantes, se retoma por 200, a la que se suman en su momento otros docentes y autoridades universitarias,

"[...] yo fui uno de los que cuando estábamos en tercer año del bachillerato diferenciado en ciencias de la salud [...] se comenzó a reunir en grupo con otros compañeros y pasamos salón por salón [...] para invitarlos a formar un grupo con la idea de proponer la fundación de la escuela de medicina [...] no recuerdo a todos los que estábamos ahí, pero éramos bastantes [...] como unos 25 o 30 [...] los que [...] comenzamos con el "borlote" de formar la escuela de medicina, se fue depurando y nada más quedamos después 12 ó 15 [...] ya hasta la fundación de la escuela [...]"..EE4

\footnotetext{
${ }^{7}$ En 1975 la Dirección de Planeación de la UNAM daba cuenta del problema de la desigual distribución de oportunidades, para los estudiantes egresados de educación media superior. Destacaban el área metropolitana de la ciudad de México, los estados de Jalisco, Nuevo León y Veracruz, como los polos con mayor migración de estudiantes. En el renglón de estudiantes que buscan un lugar en nivel licenciatura se presentaron las siguientes cifras; 5900 en 1971, 7400 en 1972, 8 200 en 1973, 6500 en 1974. A nivel nacional el estado de Nayarit se consideró, junto a Jalisco, Michoacán, San Luis Potosí, Sinaloa y Veracruz, en el grupo de prioridad 3, los cuales tenían una alta captación de estudiantes a la vez que una alta emigración.

${ }^{8}$ Un ejemplo es la fundación de la Escuela de Medicina del Instituto Autónomo de Ciencias y Tecnología (hoy Universidad Autónoma de Aguascalientes) (Barba,2000). El otro, la fundación de la Facultad de Medicina de la Universidad de Colima. (www.ucol.edu.mx)
} 
La primera acción concreta dentro de éste proceso de origen, nos remite a la formación de la comisión "Pro-construcción de la escuela de medicina",

“[...] de los que me acuerdo porque seguramente hubo más; Leopoldo Medina, Adalberto Jiménez, Ramón Silva, Mario Alcántar que en paz descanse, Benjamín Contreras, dos hermanas que hace muchísimo[...] no veo, las Tafoya [...]. De médicos que en ese tiempo se empezaron a integrar con nosotros en las pláticas, apoyarnos y orientarnos; el doctor David Trejo, el doctor Javier González Covarrubias, eran tres o cuatro médicos [...] '. EE6

Es así como junto a este grupo de alumnos pioneros, organizados y representados en la comisión, aparecen también los primeros médicos de la región, que con su entusiasmo y colaboración empiezan a apoyar algunas acciones,

“[...] el primer maestro que nos comenzó a apoyar en esto fue el doctor Alejandro López Díaz, él tenía la clase de medicina social y preventiva en la preparatoria, y él fue el que nos apoyó mucho, al menos con la emoción. No permitió que disminuyera la emoción y él nos impulsó. Al tiempo se unió el doctor David Trejo. Ellos dos fueron los que abanderaron el movimiento para la fundación de la escuela".EE7

Así, dan inicio las primeras entrevistas con las autoridades rectorales en turno, a quienes se debió de convencer en primer término de la necesidad de la apertura de una nueva escuela dentro de la universidad; en esta primera actividad la propuesta planteada por la comisión no fue ampliamente aceptada, hubo entonces que entrar a un largo proceso de convencimiento,

\footnotetext{
"Hubo cierta resistencia porque ellos argumentaban que el presupuesto universitario no era suficiente [...] ya se hablaba de que había muchos médicos en el país y que no era posible la fundación de la escuela: porque no habría lugar para el ejercicio profesional. Y nos invitaban a que cambiáramos de escuela, que tuviéramos otra vocación, pero, obviamente estábamos con el dedo en el renglón'..EE8
}

Ante esta situación, la comisión decidió encaminar sus esfuerzos por otro rumbo; convencer al presidente de la república de la necesidad de abrir una escuela de medicina en la universidad, y los aspectos positivos que traería para la población estudiantil,

"El rector tuvo que ceder, porque nosotros no estábamos dispuestos a abandonar el proyecto,
tuvimos que brincarlo. Entonces comenzamos a ser como la sombra de Luis Echeverría, a
donde sabíamos que había campaña política y que iba[...]nos aparecíamos[...] ]aquí fue la
primera vez, (que) le planteamos la necesidad de la escuela de medicina. No me acuerdo si
vino invitado por el Gobierno del Estado a inaugurar una obra[...]pero nos metimos, porque
no tuvimos un lugar en la agenda del presidente[...]le dimos un sobre y platicamos con él".EE9

De esta forma la comisión contó de manera oficial, con el apoyo de las autoridades universitarias, y se inician con mayor formalidad los primeros trabajos de gestión, “[...]Ricardo Vidal llamó a David Trajo González, que era gente de su confianza, director de la Escuela de Enfermería en 
ese tiempo, para que nos orientara y acompañara conjuntamente con Alejandro López Díaz, médico de la universidad a hacer las diferentes visitas", EE12

\section{Los gérmenes de una propuesta médica innovadora en el estado de Nayarit}

La apertura de la escuela de medicina, implicó diversas acciones. Fue un proceso paulatino que inició con pláticas entre las autoridades rectorales, entrevistas con el presidente de la república, la visita a otras escuelas de medicina de la época en la región, el establecimiento de contactos y relaciones con las autoridades de la Facultad de Medicina de la UNAM, el apoyo de CLATES (Centro Latinoamericano de Tecnología Educativa en Salud), la selección y el período de adiestramiento de los primeros tutores, la organización del primer curso propedéutico y la adopción oficial del plan de estudios. Dentro de este largo proceso, el papel del estudiante y su opinión siempre estuvieron presentes. La adopción del plan de estudios para la futura escuela, en gran medida estuvo apoyada por la decisión de los integrantes de la comisión.

Los viajes a otras escuelas de medicina, sirven para comparar los planes de estudio

La visita y estadías a diversas instituciones educativas representativas en aspectos de enseñanza de la medicina, fue una de las actividades importantes, y decisoria respecto de la selección, del plan de estudios, dadas las condiciones regionales e institucionales. De esta manera, se visitaron: la Escuela de Medicina de la Universidad de San Luis Potosí, la Escuela de Medicina de la Universidad Autónoma de Aguascalientes, la Escuela de Medicina de la Universidad de Puebla, la Escuela de Medicina en la ciudad de León Guanajuato, la Escuela de Medicina de la Universidad de Querétaro y en la Facultad de Medicina de la UNAM su Programa de Medicina General Integral A-36 y el tradicional; para conocer y convivir con alumnos y docentes de los diversos planes de estudios.

Estas visitas permiten contrastar las condiciones en que se desenvuelven los proyectos educativos novedosos, junto a los problemas cotidianos por los que atravesaba la enseñanza médica de la época: énfasis en los contenidos informativos, ambigüedad en las metas, orientación teorizante y libresca alejada de la realidad social y profesional concreta, reforzamiento de la enseñanza informativa en detrimento de un aprendizaje formativo, una evaluación aislada del proceso enseñanza-aprendizaje y encauzada a incentivar la repetición de contenidos teóricos, finalmente la separación de la docente y la investigación (Witker, citado por González, 1988). 
en el país a problemas de salud, de otras partes del país se buscó a aquellas instituciones que estaban destacadas como las más relevantes en la enseñanza de la medicina[...]e hicieron estadías los estudiantes para analizar la forma en que se enseñaba la medicina en estas instituciones. Esto llevó a la conclusión de que había un gran divorcio entre la naturaleza de los problemas de salud y la forma en que se solucionaban éstos en las instituciones de enseñanza de la medicina del país” (Entrevista, Manuel Ulloa Herrero, 1998)

Las estancias permitieron a los integrantes de la comisión, un acercamiento a la realidad cotidiana de la enseñanza en estas escuelas de medicina, e iniciar largos debates internos, sobre temas y circunstancias en ese momento cruciales para la enseñanza de la medicina; la permanente orientación biologicista en la formación de médicos, ausente de los procesos sociales en los cuales enfermaban y morían los mexicanos, la reducción a explicaciones biologicistas de los ámbitos psíquicos y sociales de la salud-enfermedad, no siendo tratados como fenómenos; colectivos, históricos y socialmente determinantes. Así como la minimización de los individuos en aspectos como: clase social, actividad productiva, sexo y grupo etáreo (Abreu, 1995). Queda constancia que de la estancia que más impactó, fue la realizada a las unidades donde se desarrollaba el (A-36) tanto en la ciudad de México como en el Estado de México: las de Cuajimalpa, Agrícola Oriental y Ciudad Nezahualcóyotl, las vivencias y experiencias han quedado plasmadas así,

\begin{abstract}
"Estuvimos varios días viendo las actividades, hablamos con los estudiantes, estuvimos en algunas clases con ellos. Nos gustó mucho la participación que tenían directamente en las comunidades, porque había mucho trabajo en comunidad. No se podía entender a la escuela de medicina, sin estar en contacto con la gente en la ciudad, en el pueblo, en las colonias y el médico. El estudiante estaba en el aula, aprendía aspectos de sociología y de desarrollo de la comunidad que era una de las materias fuertes. Regresaba a la colonia a organizarla como si fuera un promotor político, agente de cambio. Se detectaban las necesidades de la colonia, a los líderes naturales y comenzaban a hacer propuestas entre los estudiantes y los líderes. Recogían la opinión de la gente y entonces se daban a la tarea de gestionar lo necesario para resolver sus problemas básicos de salud[...]”..EE14
\end{abstract}

\title{
3 SE ENTABLAN LOS PRIMEROS CONTACTOS Y VÍNCULOS CON LA UNAM
}

Empezar a concretizar algunas de las ideas sobre el plan de estudios idóneo para Nayarit, y las tareas inmediatas a realizar, fue posible gracias a que las autoridades universitarias nayaritas en turno; el rector, el secretario general, directores y profesores universitarios, iniciaron el largo camino de gestión que iba a allanar el trabajo a desempeñar por los alumnos preparatorianos a través de la comisión, "[...]y entramos en contacto con la gente también de mucha confianza de Ricardo Vidal(rector), que era Manuel Ulloa Herrero [Quien] tenía muchas relaciones, ya estaba "muy empapado" de lo que eran los planes innovadores, los más novedosos de medicina y nos puso en contacto con gente del plan A-36[...]" EE16

Gracias a las gestiones realizadas desde Nayarit, la comisión y los docentes se entrevistan con autoridades de la Facultad de Medicina de la UNAM y del (A-36), 


\begin{abstract}
"Si, hablamos con el (director de la facultad de medicina de la UNAM), nos habló de dos corrientes de la [...] educación en medicina. Nos decía que él con su formación y no muy convencido del A-36 todavía porque lo tenía como un plan piloto. El doctor Laguna [...] decía, “-está esa opción pero ustedes ocupan cadáveres y todo para hacer disección, un hospitalescuela desde el inicio-" y [...] era una infraestructura que no teníamos aquí. Simplemente no nos imaginábamos cómo íbamos a obtener cadáveres [...] prácticamente los desconocidos eran muy pocos aquí en el estado, todo mundo nos conocíamos, cuando menos de referencia y decían, - "éste viene de Ruiz- "[...]y no había desconocidos en el Servicio Médico Forense, que era nada más una plancha de concreto que había en el sótano del Hospital Civil y nada más, entonces no había cadáveres [...] entonces el que más se acercaba por todo el ambiente social que vivíamos era el A-36"EE21
\end{abstract}

Una parte importante de este inicio de relaciones entre las dos universidades, fue la presencia del doctor Álvarez Manilla quien en ese tiempo, fungía como director de CLATES -institución universitaria que colaboraría en la formación de los primeros docentes- y secretario de Educación Médica de la Facultad de Medicina,

“(Manuel Ulloa) me hizo la invitación a nombre del grupo[...](vine) a describirles la filosofia del A-36, para eso fue a lo que me invitaron y ahí ellos manifiestan el interés de que se les asesore en implantar el plan aquí[...]cuando conocen no sé a través de qué medios que existe el A-36, me hacen una invitación a venir y hablar sobre el Plan A-36[...]yo me enteré por una invitación y ellos supieron que había esto en la Facultad de Medicina de la UNAM[...] se les dijo que sí se les podía apoyar y una de las acciones[...]fue orientar a los maestros, que consistió básicamente en enseñarles la filosofía [...]”" (Entrevista, Álvarez Manilla,2002)

\title{
4 SE OPTA POR EL PROGRAMA DE MEDICINA GENERAL INTEGRAL A-36
}

Una de las decisiones que marcaron el futuro académico de la (UAM), fue la aceptación del (A36) como el plan oficial para ella. Esta decisión estuvo sustentada en tres hechos; por un lado el análisis y evaluación realizada a los otros programas de estudio de medicina, los cuales se consideraban, “[...] un tanto alejados de nuestra realidad o por lo menos de nuestra realidad inmediata, o que requerían de crear toda una infraestructura, con la que no contábamos”.EE23

Así como las "bondades" que ofrecía el (A-36) para su puesta en marcha, ante situaciones de infraestructura y financieras que se mostraban limitadas en la universidad, y que un plan de estudios tradicional requeriría de cuantiosas inversiones,

“[...] siento que por la situación de las facilidades en cuanto a infraestructura [...] crear una escuela de medicina con un plan, que no requiriera de tanta inversión, de tanta infraestructura, de tener un hospital, grandes laboratorios, en cuestión de patología y manejo de cadáveres".EE24

Finalmente, lo que atrajo la atención del alumno, fue la filosofía, el enfoque del estudio y la enseñanza de la medicina, los que marcaban grandes diferencias respecto de los planes tradicionales de la medicina, 
"[...] habiamos analizado, visitado, estudiado diferentes planes de estudio y que nos atraía más éste nuevo enfoque de la medicina, el enfoque integral y el manejo [...] estudio de la medicina desde otro punto de vista, no biologicista [...] el contacto directo y desde un inicio con la población [...] sana o población en su medio ambiente y paulatinamente irnos integrando al contacto con el proceso salud-enfermedad[...]”.EE25

Con este hecho, los alumnos, autoridades y docentes pioneros se adelantan a las conclusiones de la Primera Reunión sobre Principios Básicos para el Desarrollo de la Educación Médica en América Latina y el Caribe ${ }^{9}$, que en el rubro de creación y funcionamiento de instituciones formadoras de médicos, resaltaba que...

"Al considerar proyectos de creación de nuevas instituciones formadora de médicos deberán favorecerse aquellos que planteen soluciones innovadoras basadas en la adecuada aplicación de conceptos técnico-científicos y pedagógicos válidos y coherentes"

\section{Inician las actividades del primer curso propedéutico}

Otras de las acciones de la comisión de alumnos junto con las autoridades universitarias, fue la organización del primer curso propedéutico para la selección de alumnos a primer año. Uno de los primeros resultados de los acuerdos establecidos con anterioridad, entre la UNAM y la UAN, “ $E l$ (Manuel Ulloa) fue uno de los que organizó el curso propedéutico con Carlos Viro, que también tuvo mucho que ver para esa idea de organizar el curso propedéutico'EE27

La implementación del curso propedéutico se desarrolló bajo circunstancias particulares; la primera es que se llevó a cabo antes de que la (UAM) contara de manera oficial con instalaciones propias, la segunda cuestión, lo representó la falta de personal médico-docente de la capital, para atender las necesidades del curso. Motivado en gran parte por el rechazo a la presencia de la naciente escuela, por lo que se contó con una minoría de médicos de la región, y se hace necesario invitar a otros docentes principalmente de aquellos que participan en la UNAM. Deben destacarse dos aspectos sobresalientes a las actividades del curso propedéutico, un primero donde se distinguen las condiciones adversas y limitantes que se encontraron dentro de la universidad para su puesta en marcha,

\footnotetext{
“En los jardines de la universidad, porque todas las escuelas (estaban) ocupadas, en ratos nos prestaban aulas en la Escuela de Enfermería o en la Escuela de Odontología, porque eran las áreas de la salud. Pero estuvimos también en la Escuela de Economía, leyes, prácticamente en todas las instalaciones de la universidad. En momentos en que estaban las aulas desocupadas y cuando no, estábamos en los jardines”. EE28
}

Lo segundo, son las nuevas orientaciones en la educación médica, entre las que destacan;

\footnotetext{
${ }^{9}$ La reunión se realizó del 12al22 de enero de 1976 en Caracas Venezuela, patrocinado por la OPS y la FEPAFEM.
} 
matemáticas, bioestadística,

\begin{abstract}
"Este programa se integró [...] con la participación de la gente de CLATES. En donde además de introducirnos en lo que era [...] el concepto adecuado, diría yo de la medicina, donde no solamente se está viendo el cuerpo humano y sus enfermedades. Sino el individuo como un conjunto, interactuando con su sociedad, en su ambiente, y de esa manera se enriqueció. Había quienes de los compañeros [...] decían ¿y esto que tiene que ver con la medicina? entonces obviamente con nuestra inexperiencia que hay que aceptarla, no lo notamos. Hasta al paso de los años, en donde nos dimos cuenta que existe un "hipervínculo"[...] al igual que todas las áreas del conocimiento”. EE30
\end{abstract}

Mientras se desarrollaba el curso propedéutico, la participación del CLATES ${ }^{10}$ en la formación del profesorado se hizo importante,

\begin{abstract}
“[...]me enteré a través de un jefe de enseñanza del IMAN que era amigo de Manuel(Ulloa)[...] dijo "-yo quiero a ese loco-", [...] ] ese loco fui yo, me dejé seducir y no me arrepiento[...] hubo un proceso de formación, porque no estaba formado para ser profesor, diseñar programas ni mucho menos[...].Un año asisto a CLATES ${ }^{11}[\ldots]$... para adquirir alguna habilidad técnica de administración educativa, de conceptualización de procesos organizativos del cuerpo docente[...] y en contacto con Carlos Viro[...]para "empaparme", vamos a decirlo así en esos términos de la ideología de este programa[...]La información que tenía del Programa de Medicina General Integral, de entrada era muy general, yo no digo vaga, pero muy general y al contacto con Viro, pude ver mejor todas las dificultades concretas, desde el problema del reclutamiento del tipo de estudiantado, el profesorado que debe ser muy pertinente para un tipo de proyecto así" (Entrevista, Guy Duval Berghman,2002)
\end{abstract}

\title{
Los primeros docentes y las muestras de rechazo a la apertura de la escuela de medicina
}

Para los programas médicos innovadores de la época, el reclutamiento de personal docente afín o idóneo a las necesidades, fue uno de los problemas cruciales por los que atravesaron. Para muchos fue el obstáculo insalvable -y aún hoy en día lo sigue siendo-, que contribuyó en gran medida a su desaparición. Para el caso nayarita, no fue la excepción, ésta cuestión fue una barrera que no pudo salvarse satisfactoriamente, en el sentido de contar con un docente idóneo (médico general, comprometido con la filosofía del programa, de tiempo completo -casi exclusivo-, no practicante de la medicina privada). Y desde los primeros momentos empezaron los problemas. La puesta en marcha del plan de estudios (A-36) pasó por etapas peculiares, una de ellas era la necesidad de contar con personal académico que cubriera aspectos pedagógicos específicos, adecuadas a un programa modular, otro, era congeniar con la filosofía de un plan de estudios, que había nacido en México en calidad de plan piloto. Una cuestión última era dar respuesta a, ¿qué este tipo de personal docente era necesario formar?, debido a que la inmensa mayoría de los médicos incorporados en las instituciones del sector

\footnotetext{
${ }^{10}$ La presencia de los Centros Latinoamericanos de Tecnología Educacional para la Salud (CLATES), uno en la ciudad de México, fue posible al copatrocinio de la OPS-UNAM-ANUIES y el segundo en Brasil con la participación de la OPS y la Universidad de Guanabara en Brasil. Dedicaron sus acciones a la capacitación de personal académico, de las escuelas de ciencias de la salud, en las áreas de actualización didáctica, evaluación, autoenseñanza, métodos audiovisuales y simulación escrita, planes de estudio y administración, que respondiera a las necesidades de la educación médica del momento. Hacia el año de 1976, CLATES México, había ofrecido cursos a un total de 1,700 asistentes. (Soberón,1991)
} 
salud, habían egresado en su mayoría de planes convencionales.

La siguiente circunstancia que se presentaba para las autoridades universitarias, fue el rechazo tácito por parte del sector médico para la apertura de la escuela de medicina, y una manera de expresarlo, fue su negativa -en la mayoría- a participar del curso propedéutico,

\begin{abstract}
"[...] hubo mucha resistencia, hubo negativa, hubo rechazo por la comunidad médica y manifestó abiertamente a decir, no a la fundación de la escuela de medicina, tuvimos que romper con ellos, ¿no quieren? bueno, pues no quieran porque obviamente era celo. Ahora lo veo hacia el pasado y era celo[...] se sentían desplazados en el futuro y es una cuestión natural, envejece uno[...] y es el círculo de la vida que ellos no lo entendían. Porque les estaba yendo muy bien, económicamente eran los grandes, eran las "vacas sagradas", eran "los intocables", ellos tenían acaparado todo el mercado. Puedo decir de la atención médica, se daban el lujo de estar donde ellos querían, operar a quién ellos querían, así, tener sus consultorios abarrotados porque no había más médicos"..EE31
\end{abstract}

De esta manera fueron pocos los docentes de la capital del estado, que participan en el curso propedéutico, la mayoría de los que apoyan las primeras actividades del curso propedéutico y en los cursos normales son invitados de la Ciudad de México,

"El doctor Toscano se integró con nosotros en el curso propedéutico y había un grupo de maestros invitados, que nos manejaban [...] cuestiones nuevas para nosotros, en cuanto a filosofía, estadística, epidemiología y cosas que nosotros no habíamos manejado en preparatoria”,EE32

\title{
Se donan las instalaciones para la escuela de medicina
}

Al término del curso propedéutico y aplicado el examen de selección de los alumnos. Uno de los puntos culminantes en este proceso lo representa la inspección-visita que se hace a diversos inmuebles de la capital del estado,

"[...] la tenía el gobierno del estado, "-ésta es la opción que tenemos aquí, ustedes busquen otras- “. Otra opción era el Hospital General de Salubridad, pero no era suficiente porque era quitar cuartos de hospital [...] de los médicos internos, que eran cuartos muy chiquitos[...]. El área que estaba fuera del hospital era también para construir, entonces no había dinero para construir, nos quedamos con esa opción: del edificio "Fenelón" [...]".EE34

A fin de ubicar las instalaciones más adecuadas, actividad en la que continúan teniendo una intervención importante las autoridades rectorales y la comisión de estudiantes...

“La “Casa Fenelón” la conseguí como rector, se la pedí a Gómez Reyes (gobernador) y el me la cedió, ahí fue la primera escuela de medicina, me tocó fundarla[...] lyo conseguí el subsidio, fui con Gómez Reyes y le dije, - "tú ya vas a salir debes dejar el subsidio-”, “-¿cuánto es?”-, son 150,000 pesos[...] y -lo autorizó-. (Entrevista, Ricardo Vidal Manzo, 1998).

\section{El inicio de las actividades}

Meses después que el Consejo General Universitario, por unanimidad aprobara la creación de la escuela, ésta abre sus puertas. Fue el lunes 17 de marzo de 1975, cuando los periódicos de la época 
daban cuenta del acontecimiento,

\begin{abstract}
"En acto que intentó ser solemne, ayer el Rector de la Universidad de Nayarit declaró inaugurada la Escuela de Medicina, culminando así con una aspiración tanto del Gobierno del Estado como de las propias autoridades de la Universidad, de crear este plantel que viene a dar la más alta categoría a nuestra Alma Mater. En el acto estuvo presente el Director del Plantel, doctor David Trejo González, y el Secretario General de la Universidad, Lic. Héctor Béjar Fonseca[...]miembros del Consejo General Universitario y decenas de estudiantes". (Periódico el Nayar, 1975)
\end{abstract}

La estructura administrativa que conformó el primer grupo directivo fue: en la Dirección el doctor David Trejo González, la Subdirección Académica con el doctor Javier González Covarrubias y la Subdirección Administrativa con el doctor Rafael Rivera Montero. Entre los primeros departamentos estaban; Contabilidad con el contador Bernardo, Sección Escolar con Celia Betancourt Pérez, Francisca Becerra y Ma. Consuelo Ulloa, Coordinación de Niveles, Imprenta con José Gómez Navarrete y Programación. Posteriormente aparecen los departamentos de Medicina Preventiva y Social, Apoyo al Aula, Evaluación, Exámenes Profesionales, Internado, Servicio Social y Taller de Fotografía.

Los tutores fundadores fueron los médicos y médicas: Pura López Cortés, Guy Duval Berhmann, Luis Téllez, Francisco Herrera Rodríguez, Arcelia González Covarrubias (f), Carlos Martínez Sánchez, Francisco Millán y el fisiólogo Alberto Núñez. Se integraron posteriormente el Ing. Paolo Roberto Vasconcelos Costa, Maximino Severiano Castillo, Maximino Arce (f) y los técnicos: Pedro Aguiar García, Víctor Casas Martínez, Juan Manuel Miramontes y Julio Mu Flores. Este logro representa el fruto de esfuerzos que meses antes habían iniciado. Para la universidad encarna un logro importante, en apoyo de la comunidad estudiantil nayarita, al ofrecer una nueva opción educativa a los jóvenes egresados del nivel medio superior,

"[...] no recuerdo detalles de la cuestión del edificio, pero repito con el apoyo de estas gentes y del rector, que incluso muchas veces las reuniones eran en su casa, juntas de trabajo[...]llegó un buen día que nos dijeron esa iba a ser nuestra escuela y por ahí circula la famosa fotografía del primer día[...] ]que llegamos a ese edificio, tomado por toda la "bola" en la calle Lerdo, antes de entrar al edificio: ese fue nuestro primer día que pisamos ese edificio, la famosa "Casa Fenelón”.EE35

Sin embargo, con la entrega de la "Casa Fenelón" no terminan las necesidades, viene una etapa de acondicionamiento en infraestructura la cual se va cubriendo en forma paulatina, con la participación del CAPCE,

"En ese tiempo no estaba ocupada, estaba en ruinas, estaba abandonada. Las puertas apolilladas, todo despintando, feo en su aspecto. Pero como traíamos un romanticismo tremendo, lo vimos hermoso y "- ¿esto va a ser la escuela de medicina?-", lo ligamos con la medicina antigua y nos sentimos caballeros de aquellas épocas, de principios de siglo. Porque 
el ambiente de la casa así se prestaba, para que fuera de principios de siglo, nos imaginábamos de batas blancas, ahí haciendo disecciones en animales. $Y$ se consiguió, no fue muy difícil que nos otorgaran el edificio". EE36

Esta situación limitante se expresó en diversas esferas de la vida académica de la escuela, sus aulas, laboratorios y biblioteca. En estos primeros días las necesidades rebasaban los precarios recursos con los que se contaba, a fin de atender a los primeros cinco grupos. En esta fase el papel de los estudiantes promotores fue significativa,

\begin{abstract}
"Simultáneamente fuimos integrando algunos equipos, fotocopiadora, material bibliográfico[...]fuimos a recoger[...] algunos equipos "usadones”, equipo nuevo incluso, para poderlo poner a la brevedad ¿no?. Inicialmente algunos de los compañeros de la comisión, recordamos, nos movilizábamos en el vehículo, íbamos de compras, le dábamos las cuentas (al director o al rector), en aquel tiempo, dado que no existía una estructura adecuada en la universidad en ese tiempo, para poder abastecer de insumos la Escuela de Medicina. Fue un trabajo realmente constante, hasta que finalmente las autoridades de la escuela tomaron la batuta y ya no fue necesario que participáramos [...] tan activamente”,EE37
\end{abstract}

El edificio tuvo que irse acondicionando de acuerdo a su población escolar, el inicio de las actividades se expresan de la siguiente manera,

“[...]entonces trabajamos en lo que era la parte principal del edificio[...] se utilizó solamente la parte anterior, en la parte de arriba al fondo se ubicaron las oficinas administrativas y [...](al) fondo del pasillo, lo que era una especie de biblioteca[...]porque nos enviaban todos los documentos "módulos", los famosos documentos y ahí se adaptó un pequeño espacio para (su) distribución. [...] el resto de la planta alta se utilizó inicialmente como aulas, después se convirtió en el laboratorio, uno de química, y uno de fisiología[...]pero inicialmente esas fueron nuestras aulas las de arriba y abajo a la entrada, atrás de la escalera era otra[...]de la entrada del cancel a la izquierda también se acondicionó para otra aula", EE40

También se acondicionaron los laboratorios

"Creo que hasta como un año después [...] llegaron el resto de los maestros[...]se acondicionaron los laboratorios, entonces pasamos a ocupar como aulas la parte de atrás del edificio", EE42

¿Y la biblioteca?

"Contamos con una biblioteca chica, no muy bien equipada[...] contábamos con una persona, que si requeríamos un libro,[...] hacia la solicitud para que adquiriéramos ese libro[...] en el primer año no teníamos biblioteca, en el segundo ya tuvimos[...],EE44

Estas vivencias contrastan con otra de las recomendaciones adoptadas por los asistentes entre ellos el Dr. Carlos Campillo Sains, quien era Subsecretario de Asistencia, de la Secretaría de Salubridad y Asistencia, participante por México- a la $1^{a}$. Reunión sobre Principios Básicos para el Desarrollo de la Educación Médica en la América Latina y el Caribe, que para la... “creación de una 
nueva institución formadora de médicos sólo deberá ser autorizada previa comprobación de que el proyecto se conforma a las políticas de recursos humanos para la salud vigentes y reúne las condiciones indispensables para un funcionamiento eficiente".

\section{Los ejes curriculares, su implementación}

a) Las nuevas propuestas pedagógicas al interior del proceso enseñanza-aprendizaje.

De acuerdo a los diseñadores del plan, la presencia de un médico general durante las 40 semanas efectivas de trabajo con el estudiante, serviría de contrapeso para contrarrestar la influencia e impacto que pudiera ejercer, la medicina de especialidad. En este sentido el tutor aparecía como la figura que tenía una participación directa en todas las actividades de enseñanza-aprendizaje, lo que le permitía realizar una enseñanza personalizada, detectando las áreas deficientes.

Se concebía al tutor como un médico capaz del manejo amplio y adecuado de las áreas médicas, destacando su habilidad para relacionar y articular conceptos en su aplicación y compromiso para con el autoaprendizaje. Adicionalmente, las actividades en aula permitían la presencia de docentes invitados a fin de ampliar y profundizar contenidos específicos, los que se combinaban con búsqueda bibliográfica, discusiones grupales, revisión de experiencias en clínica, y comunidad, y la presentación de sociodramas y prácticas equivalentes y análogas (García Colorado, 1993).

Para el (A-36) el tutor fue concebido como un modelo de práctica de la medicina general, el cual coordinaba parcial o totalmente, diversas actividades del plan de estudios, en las áreas de: comunidad, clínica, aula, laboratorio. Debiendo considerar al módulo como la estructura didáctica multidisciplinaria que permitiría integrar en cada actividad áulica las áreas de: biología, psicología, sociología, epidemiología, patología y clínica. Aunado a ello la aplicación de una metodología activa que permitiría al alumno desarrollar actividades como: práctica clínica en los servicios asignados, estudio individual en los materiales de autoenseñanza, libros de texto y bibliografía complementaria, prácticas de laboratorio, sesiones clínicas, seminarios integradores, sesiones bibliográficas, entre otros.

Las actividades áulicas representan el núcleo central del PMGI A-36, era un sello de distinción respecto de los programas de medicina tradicionales. La nueva práctica docente médica, se volvía también, al igual que las actividades en comunidad, en una nueva actividad dentro del ejercicio docente universitario nayarita. Aún cuando las actividades académicas iniciaron en un ambiente marcado por las limitaciones, ello no fue un impedimento para la puesta en marcha del A-36. Situación que se 
combinaba con el ánimo del docente y el entusiasmo que empezaron a imprimir cada uno de los alumnos,

\begin{abstract}
"Lo que se marcó desde un principio, es que esto no era lo tradicional, a lo que estábamos acostumbrados. El maestro está al frente y nos da toda una cátedra, sino que teníamos conocimiento de cuál era el módulo que íbamos a llevar, cuáles eran los objetivos que íbamos a cumplir, qué actividades teníamos y se hacía una distribución por equipos. El mentado Phillips 66, desde un inicio empezamos a trabajar por grupos, por equipos y se distribuían las actividades del módulo para la presentación y discusión de los diferentes temas. Y bueno, coordinador por el tutor, y generalmente se echaba-el (Dr. Guy Duval) siempre ha sido bien rollero- su rollo inicial y luego ya trabajaba el grupo; discutía, analizaba y luego él hacía su cierre o su regaño o su "jalón de orejas". Porque muchas veces nos llegó a poner como "campeones" - "y éste tema no sirvió de nada”-. Pero generalmente así era, muy participativo[...] no era la cuestión de vamos, nos sentamos y escuchamos. Desde un inicio empezamos a ser nosotros, como alumnos, los que manejábamos las actividades académicas". EE46
\end{abstract}

Las actividades del tutor en aula destacaban su presencia en una gama diversa de nuevas actividades; la asesoría intra-extramuros, participación en las actividades comunitarias, presencia en la evaluación parcial y final de las actividades, coordinación de las actividades en clínica, dar una mayor participación al alumno en la presentación y discusión de los diversos temas, lo que colocaba la relación del alumno-tutor en una igualdad, es decir, se hablaría de una mayor horizontalidad en la relación ${ }^{12}$. Este aspecto, tuvo una contribución importante en la creación del espíritu colectivo que fue incorporando al alumnado. Un ambiente de trabajo, de nuevas relaciones con el que se llegó a identificar a la (UAM) y a sus estudiantes, que posteriormente retribuyó algunos aspectos importantes del perfil profesional que buscaba el programa de estudios; como modelar una actitud de crítica y lucha de y hacia la colectividad,

\begin{abstract}
"En aula era realmente una actividad muy agradable, pero además de que los tutores en general tengo entendido de aquel tiempo, nos daban mucha confianza [...]. Nosotros hacíamos la revisión modular, que obviamente nos ayudó mucho la UNAM[...] una vez [...] ]avanzado el módulo, teníamos asesorías que realmente eran auténticas asesorías con el tutor, le decíamos - "tengo ésta duda, en este aspecto no lo entiendo"-, y de esa manera nos aclaraban bastantes dudas. Nos recomendaban lecturas adicionales, algunos textos[...] '..EE47
\end{abstract}

Se va conformando un ambiente estudiantil inédito, que nutrió el espíritu de las primeras generaciones

La participación del alumnado en la creación de la (UAM) y sus signos de combatividad y persistencia, encontraron en el ambiente áulico, un crisol que lo forjó y fortaleció; en la medida que la

\footnotetext{
${ }^{12}$ De acuerdo al programa, las funciones del tutor en el primer nivel ( $1^{\circ}$. y $2^{\circ}$. años), se cubrían a tiempo completo durante 40 semanas. Se encargaba de guiar, asesorar, orientar, supervisar, conducir y evaluar el proceso enseñanza-aprendizaje. Conducía los aspectos teórico-prácticos del aula-laboratorio-comunidad, era el supervisor del adiestramiento que recibía el alumno en los espacios clínicos. Además, se encargaba de la evaluación formativa durante todo el proceso, aparte de los exámenes quincenales. (Proyecto de Readecuación curricular, 1988).
} 
relación tutor-alumno permitió una mayor apertura en la comunicación y trato. Se fue conformando un ambiente escolar interno, que no se respiraba en ningún otro espacio universitario. Este ambiente escolar, se nutrió del trabajo individual y colectivo que desplegaron los tutores, tanto dentro como fuera del plantel. Las nuevas dinámicas de trabajo académico con el estudiante y los nuevos papeles de asignación de responsabilidad, pueden dar cuenta de ello,

“[...]llegábamos -en lo personal me gustaba mucho la escuela de medicina, la "Casa Fenelón”, era un ámbito muy propio-, a las seis de la mañana y me encontraba con algunos de los tutores, pero tutores que todavía seguían con ese espíritu. Me encontraba con algunos compañeros y empezábamos a compartir información. Eran las ocho de la mañana que iniciaban las clases y nosotros ya habíamos hecho una discusión preliminar de lo que íbamos a observar en aula. Compartíamos nuestras notas, teníamos la posibilidad hasta de apoyarnos en esto que era tan difícil, lo de laboratorios. Para nosotros hasta que nos pusieran a dibujar un mar era dificilísimo ¿no?, rompieron con muchas de nuestras debilidades".EE48

Esta relación distinta entre docente y estudiante, que se iba construyendo paulatinamente, tuvo una contribución importante en la conformación del espíritu colectivo del alumnado, que fue muy importante en los siguientes años, para la existencia misma de la escuela,

"[... Jy una de las cosas que se vio, que éste plan funcionaba, porque había el gran interés de los alumnos, de cada vez preparar mejor (los) temas que se les dejaban. Porque teníamos esa gran responsabilidad y así lo pensábamos nosotros, tratar de hacer mejor las cosas. En condiciones en las que nos encontrábamos, de los recursos materiales con los que se contaba. $Y$ el único recurso que teníamos en el aula no era más que el pizarrón, el maestro y la participación de los alumnos. No teníamos más apoyo”. EE49

La construcción de este ambiente estudiantil, fue una plataforma importante, una protección que ayudó de forma particular los nuevos retos que enfrentaron los estudiantes. En esta construcción se advierte diáfanamente, la importancia del trabajo docente, la convivencia y relación entre los docentes, que marcó el trabajo académico,

\footnotetext{
"Fue muy rico el primer año, tuvimos severas complicaciones y recuerdo que en una ocasión estábamos "trabados" en genética y lo recuerdo por lo "duro" de la genética y dado que estaba la doctora Pura[... ]gentil como era, se dispuso a darnos un curso extraordinario sobre genética y fue otra cosa. Fue despertar a la posibilidad de encontrar que no todo individuo conserva todo el conocimiento, o tiene habilidades para todo. Arcelia se supo acercar a gentes de este tipo y solicitaba los apoyos. Entre ellos no había resistencias, era una comunicación impresionante y a partir de entonces, nosotros nos abrimos, porque sentíamos que estábamos muy separados de los de segundo. En ese instante cuando llega Pura y rompe con esos esquemas, nos integramos, ya no como los de primero y segundo año, no, como la Escuela de Medicina. Yo creo que fue el detonante para dar una cultura entre nosotros muy importante, fuera de egoísmos, fuera de tantas tonteras y dedicarnos más a ser los alumnos de la Escuela de Medicina, impresionante el primer año". EE50
}

En síntesis, se conforma un ambiente con nuevas expresiones de convivencia, donde los diversos grupos comparten a distintos niveles la opinión de, 
“[...] aquel grupo donde éramos más que compañeros, éramos íntimos, éramos prácticamente familiares. Porque nos veíamos mañana, tarde y noche, compartiendo no secretos, sino sueños. Compartiendo [...] nuestros de-sueños, nuestros desvelos y nos protegíamos perfectamente”. EE51

\section{b) La docencia, el servicio y la investigación}

Este eje curricular distinguía al (A-36) de los planes de estudio por asignatura en varios aspectos: uno, el aplicar la metodología de la estructura diacrónica (el proceso de aprendizaje debe progresar de lo simple a lo complejo), con la sincrónica (mantener una relación estrecha entre la información teórica y la práctica), que lo convertía en área fundamental para la formación de los estudiantes durante los cuatro años de la carrera.

Particularmente cuando ésta se encaminaba a los niveles de atención: primaria (es la atención médica primaria y de la población sana con riesgo de enfermedad), secundaria (atención a pacientes ambulatorios y hospitalización a nivel de especialidad), terciaria (atención a pacientes con padecimientos a nivel de subespecialidad).

Las diversas actividades correspondientes al primer nivel (primero y segundo años) tienen una relación con actividades de adiestramiento en el centro de salud,

\footnotetext{
“...dos meses después de ingresar, el alumno inicia su práctica en el Centro de Salud, que dura los 2 años del primer nivel, en esta actividad el alumno cumple objetivos tanto en el área cognoscitiva, psicomotriz y afectiva. La carga es de 10 horas semanales y en forma rotatoria se distribuyen los servicios de la Unidad. Es importante señalar la participación de los alumnos en los programas de inmunización, detección de enfermedades transmisibles y las pláticas de educación para la salud" (UNAM, 1978), de esta forma el alumno y
}

su tutor forman una unidad docente-asistencial, participando en la consulta externa de los servicios de Medicina Interna, Gineco-Obstetricia, Pediatría, Planificación Familiar, Medicina Preventiva.

El programa original, estipulaba que los alumnos desde los primeros años, tuvieran una participación directa en los espacios clínicos (2 o 3 veces por semana). Lo que originaba que se estuviera en un contacto cotidiano, con los responsables en los centros hospitalarios, a fin de llevar los controles y seguimientos del alumno, en cada uno de los servicios por los que rotaba. La limitante era que en el espacio clínico no se tenía una clara definición del perfil del docente clínico (García Colorado, 1993).

De acuerdo al plan de estudios, éste tipo de labores académicas marcaban abismales diferencias en el proceso de enseñanza de la medicina, respecto de los planes convencionales. En primer lugar, porque se marcaba como un gran acierto pedagógico, que el alumno tuviera un acercamiento temprano 
y permanente con la convivencia clínica. Segundo se tendían puentes de comunicación a fin de que los tutores en aula y los médicos en las clínicas y hospitales, atendieran objetivos de enseñanza-aprendizaje comunes. Tercero, con ello romper el largo divorcio que habían edificado los planes de estudio convencionales, entre la teoría y la práctica, teniendo presente la diacronía ${ }^{13}$ como principio de actividad. En cuarto lugar, éste acercamiento del alumno, le daría la oportunidad para conocer y reconocer la patología común que presentaba la población y a la vez permitiría que el alumno conociera las actividades cotidianas que realiza, tanto el médico general y el especialista, en su ámbito de trabajo: su trato y comunicación con y hacia el paciente, expresadas en sus indicaciones, procedimientos de revisión-auscultación y en su presencia física.

\section{La realidad nayarita se mostró distinta}

El acercamiento a los campos clínicos de las instituciones del sector salud, se dio de manera paulatina pasando por las actividades comunitarias, los espacios del Centro de Salud en sus servicios de Medicina Preventiva, Planificación Familiar, Trabajo Social,

\footnotetext{
"Ya en el segundo semestre nos enviaron a prácticas al Centro de Salud "Juan Escutia", estaban los cuatro grupos por equipos[...] que conociéramos cómo funcionaba el centro de salud y los diferentes departamentos: estadística, medicina preventiva, en los consultorios[...] todos divididos en equipos". EE53
}

\section{Un hecho inédito en la enseñanza médica superior, el fenómeno del rechazo del alumno}

Durante el año de 1975, las autoridades universitarias en voz del ingeniero Ricardo Vidal Manzo, el doctor David Trejo González como director de la escuela de medicina y las autoridades del IMSS e ISSSTE, firman los primeros convenios ${ }^{14}$ para apoyar las actividades de enseñanza de las(os) estudiantes en sus espacios hospitalarios de acuerdo a normas y criterios previamente establecidos. El programa establecía la presencia del estudiante en los servicios de atención médica de primer nivel y en la comunidad, por lo que era necesario contar con las instalaciones de las instituciones del sector salud, de esta manera el convenio destaca uno de sus principales propósitos “...formar un médico general integral, capaz de ejercer una medicina científica, para aprovechar las tecnologías médicas modernas, a la vez de generar las actitudes necesarias para cumplir con su nuevo papel en la

\footnotetext{
${ }^{13}$ Se entiende por ello a mantener una relación estrecha entre los conocimientos teóricos y la práctica.

${ }^{14}$ Los convenios firmados estipulan dentro de sus cláusulas; que la institución del sector salud brindará las facilidades necesarias para el desarrollo de las actividades de enseñanza e investigación. La universidad otorgará los nombramientos al personal médico que colabore en actividades de docencia, investigación y apoyo, de acuerdo a los reglamentos universitarios en vigor. La institución universitaria observará el uso adecuado de las instalaciones en equipos, horarios y condiciones. Finalmente, la escuela acatará los reglamentos y disposiciones de trabajo de la institución.
} 
comunidad urbana marginal y en las comunidades rurales del país”, para que contribuya a que la (UAM), forme “....médicos de comunidad, sobre la base de una integración plena de la teoría y la práctica a todos los niveles de enseñanza, que responda al objetivo básico de incorporar a la totalidad de la población al sistema nacional de salud, que coincida con el objetivo central del Plan Nacional". (Convenios celebrados entre la Universidad Autónoma de Nayarit y el IMSS e ISSSTE, 1975)

Sin embargo, al igual que en el caso de la apertura del tercer año de la Escuela de Derecho, donde la Barra de Abogados de Nayarit estaba en desacuerdo. La (UAM) revivió una experiencia similar, al enfrentar a los grupos de médicos de la región, que se opusieron tanto a su apertura, como a la presencia del alumno de segundo año en los espacios clínicos y de consulta externa, a recibir su enseñanza. Con ello, se inauguraban las relaciones entre la naciente (UAM) y las instituciones del sector salud. El sello que distinguió a estas prácticas, estuvo en el signo de rechazo hacia la presencia del alumno, por parte de los médicos,

“[...] porque si te puedo decir, que la comunidad médica del estado, en general, no puedo marcar porcentajes, pero creo un $80 \%$, percibíamos que estaban en contra y sabíamos y conocíamos de comentarios en contra de la creación de la escuela. Pero hubo un grupo, que sí desde un inicio [...] empezó a participar con nosotros, desde el curso propedéutico; el doctor Toscano, [...]Javier, David Trejo, López Díaz. Fueron de los primeritos [...] que se aventaban los viajecitos con nosotros y las desveladas y mal comidas [...] '. EE54

Las expresiones del rechazo.

Si las actividades en comunidad enfrentaban problemáticas diversas y se hacían cambios respecto al programa original, por ejemplo ante el número reducido de centros de salud que las apoyaran. Las actividades en los campos clínicos no ofrecían un panorama halagüeño, aquí durante los próximos siete años, serían los alumnos quienes recibirían las muestras de rechazo, ante su presencia por parte del personal médico,

"[...]porque los maestros de los campos clínicos, por actitudes políticas de extrema imbecilidad, que son las que privan en el medio ratonero que tenemos aquí, le declararon la guerra a la escuela y una buena porción, incluyendo a algunos que todavía están en la escuela $e$ incluyendo a algunos que han sido directores[...]gente que desde el principio tuvieron una actitud y yo creo que siguen teniendo una actitud muy belicosa en contra de la escuela[...]", EE55

Esta sensación de rechazo que sufrieron la mayoría de los alumnos, quedó plasmada en los siguientes comentarios cotidianos, en los que se destaca por un lado; la no atención hacia el alumno y la hostilidad:

“- así que vienes tú, de la escuelita de medicina," 
“-¿qué andan haciendo allí, no sirve”,

“-mediquitos”,

"-doctorcitos, de la escuelita",

“-¿van a ser médicos descalzos, verdad muchachos?”

“- ¿a poco ustedes si son estudiantes de medicina?”

“-ese es el plan, que los hagan a ustedes enfermeros, así muy bien preparados, pero enfermeros,",

“-¿para qué te mandaron aquí?”

“-qué íbamos a ser médicos comunitarios y que solamente íbamos a recetar plantas [...] ]a curar con plantas, porque no íbamos a saber curar"

“-de un A-36, con eso no van a vivir jamás en la vida”

“-ibamos de estorbantes"

"-ustedes nunca van a ser competencia para nosotros"

"-si te quieres ir, yo te firmo"

“-si quieres te firmo y ya te puedes retirar".

Esta actitud de rechazo para el alumno, hizo que la propuesta original del programa tuviera diversos cambios; el primero, era que se veía afectada la coordinación, con las actividades en aula, lo que se conocía como ( sincronía $^{15}$ ), un segundo punto, que incluso con la presencia de un programa académico de actividades, este no hubiera podido supervisarse, en tercer lugar, la presencia del tutor fue en un inicio esporádica, para terminar ausentándose definitivamente a mediados del segundo año de actividades.

De esta manera, la propuesta pedagógica en clínica empezó a resquebrajarse, mostrando la enseñanza diversas situaciones inéditas e inverosímiles,

"[...] cuando llegamos al Seguro (Social) a hacer prácticas, inmediatamente, fueron poquitos los que nos aceptaron. Empezamos a sentir ese ambiente hosco, incluso había compañeros que te decían: - "a mí me tocó suerte”-, otros compañeros decían, que eran completamente ignorados por los médicos y aparte sarcásticos, porque decían que no íbamos a ser médicos, cuando egresáramos[...] ’'EE57

La relación docente-estudiante y el seguimiento de los objetivos educacionales, no ayudaron a reconstruir ésta deteriorada relación. Llama mucho la atención, que la forma de rechazo, en el nivel de

\footnotetext{
${ }^{15}$ Se entiende como estructura sincrónica, “[...]al principio pedagógico de mantener la información teórica incorporada a la práctica, se debe contemplar el adiestramiento en servicio, que en caso de los alumnos del Programa de Medicina General Integral, se da en los Centros de Salud, Unidades de Medicina Familiar, Hospitales Generales y Hospitales de GinecoObstetricia”. (UNAM, 1988).
} 
enseñanza médica, se haya circunscrito al papel de pasividad que por mucho tiempo mantuvieron los estudiantes,

\begin{abstract}
"Con el doctor Chilo y [...] el doctor Rojo, lo que hacíamos era observar lo de la consulta, por un lado, ahi "estorbando" en los cubículos al médico; viendo como exploraba y posteriormente ya nos ponían [...] a hacer exploración. Exploraba él y a veces (porque tenía un montón de consulta), me daba la oportunidad de que yo explorara. Poco a poco me enseñó a hacer[...]las historias clínicas, (el) [...] interrogatorio”.EE58
\end{abstract}

El área de consulta externa fue considerado desde un inicio el espacio idóneo para la enseñanza de la medicina que el estudiante requería, por eso, aunque no fue el único, si fue el que dejó más grabado en el recuerdo colectivo del estudiante, el trato y su relación con el médico general o especialista,

"[...]llegábamos a clínica y durante muchos años nos tenían sentaditos, la mayoría, lo digo, la mayoría de los médicos que teníamos en clínica, nos trataron peor que suela de zapato. Únicamente les servíamos para hacer las recetas y las notas. Porque tenían una letra pésima y nosotros empezamos a adquirir habilidades para hacer notas clínicas muy rápidas y muy completas. Tuvimos un privilegio muy grande de poder sintetizar las situaciones en los primeros años y eso nos dio una habilidad, para cuando llegamos a clínica y creo que esas buenas notas, por ahí deberán de estar en más de un expediente”.EE59

Una siguiente área clínica de capital importancia, lo constituyó el quirófano, en éste el rechazo tuvo una expresión distinta,

\begin{abstract}
"Recuerdo que fue entonces, cuando nos decían "los pollitos", porque teníamos que ir a quirófano de amarillo. Muy señalados, extremadamente señalados, ninguneados, sobajados. En unos quirófanos, recuerdo bien, nos tenían pegados a la pared y desde la pared querían que nosotros estuviéramos aprendiendo". EE60
\end{abstract}

Esta actitud hostil que de manera cotidiana enfrentó el alumno de las dos primeras generaciones, provocó una reacción que hizo resurgir el sentimiento de unidad y solidaridad entre el alumnado. Empezó una manifestación colectiva de enfrentar éste contexto adverso, como una respuesta natural de un alumno que ha convivido y ha luchado antes y después del nacimiento de la escuela de medicina.

No puede dejar de mencionarse, que en este inmenso desierto de desatenciones y hostilidades, los alumnos encontraron, la disposición, el apoyo y la buena voluntad de algunos médicos,

\footnotetext{
"[...] el doctor Herrera, creo que desde el curso propedéutico nos dio clases, fue de los más "antigüitos", que trabajaron con nosotros y fue también de las gentes que nos apoyaba. El trabajaba en la Secretaría de Salud, en ese entonces en el Centro de Salud y fue con el primero que empezamos a tener peso. Con el radiólogo Plata, que incluso nos dejaba "meter mano" y nos explicaba todo [...] hubo gente que nos abrió las puertas y que nos empezó a dar apertura y a enseñar [...]"..EE61
}

En Tepic los alumnos son vistos como rivales en la actividad profesional de los médicos 
La presencia de una nueva institución educativa universitaria, motivaba a una serie de cambios y transformaciones futuras del mercado laboral de la región. Para esos momentos la mayoría de los profesionales médicos tepicenses eran egresados de las facultades de medicina de la Universidad de Guadalajara (UdeG), Universidad Autónoma de Guadalajara (UAG) y la UNAM, estos primeros médicos empezaron a formar su clientela tanto de la capital Tepic, como del interior del estado, tanto por su calidad profesional, precio de la consulta, trato personal-profesional, la acumulación de un determinado capital y la conformación de grupos profesionales médicos que controlaban determinada área y población, etc.. Por lo que las muestras de desatención a los alumnos en su preparación clínica, fueron muestra de un inminente resquebrajamiento de esta presencia,

\begin{abstract}
“Después lo comprendí, ellos eran los dominadores del ámbito médico. No tenían competencia y nosotros éramos la competencia. Yo no puedo estar dándole de comer a quien después me va a venir a desplazar, refutaban nuestros documentos, aún sin sustento técnico. Cuando les decíamos, -mire doctor es que ésta es copia de Nelson, nosotros no tenemos para comprar libros, pero es el mismo libro que usted tiene-, se quedaban de a seis, ¿verdad?, sí pero tuvimos que romper muchisimas barreras"..EE62
\end{abstract}

\title{
c) Trabajo de comunidad
}

Para el (A-36) las actividades comunitarias, junto a la participación activa en aula-laboratorios y el adiestramiento clínico, representaban una de sus áreas fundamentales, a la vez, que una de sus características principales, dentro del proceso de enseñanza-aprendizaje de la medicina. En cierto sentido era uno de sus sellos distintivos respecto de planes de estudio tradicionales. De acuerdo al programa, dos propósitos se buscaban; primero que el alumno conociera y entrara en contacto con la realidad médico-social a fin de lograr médicos muy familiarizados con los aspectos preventivos y curativos respecto de los problemas de salud más frecuentes en el medio. Y segundo que el alumno aprendiera a trabajar dentro de un equipo de salud junto y con la participación de su comunidad (Vega,1990).

Durante el primer año, el alumno desarrolla el diagnóstico de la comunidad a su cargo y efectúa el seguimiento de familias, en el segundo año; realiza diversos subprogramas: Detección Defectos Posturales, Salud Bucal dentro del programa de Higiene Escolar en una escuela primaria e investigaciones en Salud Pública. Las actividades de ésta naturaleza, permitían al estudiante un contacto inicial con las problemáticas de salud-enfermedad, cotidianas de la comunidad en cuestión. El programa ponía al alcance del alumno, el contrastar una explicación biologicista de la enfermedad, frente a una explicación más multifactorial respecto de las causas y orígenes de los padecimientos más frecuentes. 
Por una exigencia misma del programa, las áreas seleccionadas eran colonias de la periferia, que carecían de los servicios públicos indispensables (alumbrado, pavimentación, drenaje, agua potable), su población trabajadora se distinguían por ser obreros de la industria, empleados domésticos, trabajadores temporales, que los distinguían por ser económicamente vulnerables. Ante éste panorama el estudiante estaba en contacto con circunstancias sociales, muchas veces ajenas a su vivir cotidiano, lo que le daba la oportunidad de contrastar opiniones y puntos de vista, tanto de las posibles causas de morbi-mortalidad, como de las soluciones idóneas y posibles, de acuerdo a las circunstancias y recursos de la comunidad.

Las actividades comunitarias -se contemplaba- apoyaban la estructura diacrónica y sincrónica del programa; respecto de la primera, representaban desde el primer año, los cimientos y el primer eslabón ${ }^{16}$ que tenía el alumno a cubrir, para acercarse de manera paulatina dentro del espacio clínico, a la consulta de la medicina de especialidad. En el segundo año, las actividades se encaminaban al área de Higiene Escolar, donde el alumno tenía una participación importante dentro de una escuela primaria. Respecto de la estructura sincrónica, se consideraba que los espacios donde se desarrollaban las actividades comunitarias, eran propicios para establecer acercamientos entre las temáticas teóricas y prácticas.

El trabajo comunitario, representaba un espacio educativo en el cual se podrían desarrollar; funciones de promoción de la salud, reconocimiento de factores de riesgo a nivel individual, familiar y comunitario,

\begin{abstract}
"se buscaba transformar la visión dominante de enfoque curativo, por una postura anticipatoria que reconoce que la enfermedad es generada por condiciones de tipo social, económico, psicológico y biológico que al ser detectadas antes de que aparezca la enfermedad, reduce los daños físico y psíquico, el sufrimiento y los costos derivados de la atención médica", a la vez "... a través del contacto con este tipo de población, el alumno puede aprender las medidas preventivas que formarán parte de su capacidad como médico general" (Álvarez Manilla, citado por García Colorado, 1993:67, UNAM, 1978).
\end{abstract}

Con las actividades en comunidad de la (UAM) se inauguran dentro de la universidad nayarita, un tipo de acciones curriculares, que anteriormente no habían tenido espacio, ni se les había dado gran importancia. Las escuelas de Agricultura, ingeniería Pesquera, Medicina Veterinaria y Zootecnia y Economía, de manera paulatina empezaron a destinar espacios importantes en sus programas académicos, que se tradujeron en un número considerable de horas teórico-prácticas, lo que de alguna manera revolucionaba no solamente el quehacer del docente y del estudiante, sino penetraba a los aspectos filosóficos, sociales, políticos, educativos, y de perfil profesional de los egresados.

\footnotetext{
${ }^{16}$ Dentro de esta cadena, podemos ubicar a la comunidad como el eslabón inicial, seguido por la consulta en el centro de salud, la consulta en la unidad de medicina familiar y la consulta de las especialidades médicas.
} 
Las actividades en comunidad. Una experiencia educativa política, inédita en la universidad

La (UAM) inicia sus actividades comunitarias en el año de 1975, con la selección de las colonias: Morelos, Heriberto Casas, El Rodeo, Moctezuma, y algunos de los ejidos dedicados a la producción de caña de azúcar, circundantes a la capital. Donde se organizaron y llevaron a cabo diversas acciones; levantamiento censal por manzanas, encuesta de necesidades, estudio socioeconómico, identificación de los líderes naturales,

\begin{abstract}
"Fue primero un recorrer Tepic, de todos los alumnos de la escuela, incluso de los tutores para semblantear o al menos en lo que se veía lo macro, como estaban las condiciones de las colonias y entonces hicimos una lista de prioridades con la colonia fulana, sutana, la colonia Versalles ni tocarla, ni la San Juan vivían los puros ricos y nos enfocamos hacia las colonias populares. Sobre todo las que ya estaban más o menos establecidas para que no nos diera mucha dificultad [...] había mucha exigencia porque algunas gentes comenzaban a decir, bueno pues no hay agua, no hay drenaje, no hay luz- y el gobierno así como a ignorarlos". EE65
\end{abstract}

Este tipo de actividades resultaron ser pioneras en su tipo y para los miembros de la primera generación, la experiencia resultó ser altamente significativa. Por primera vez la (UAM) se hacía presente ante los grupos más vulnerables, tratando de estudiar y conocer su realidad médico-social, mostrándose como un gran laboratorio social,

\begin{abstract}
"recuerdo cuando nos llevaron a campo... ¿verdad? Que nos llevan a un campo cañero y nos ponen a cortar caña, -un choque por Dios, un choque, ¿Cómo el médico de batita blanca y zapatito blanco se va a tiznar?, -para entender la realidad del cañero-. Fue impresionante, fue un choque [...] perfectamente medido por ustedes, mas sin embargo, con unos resultados fabulosos para muchos de nosotros: fue el cambio. El poder penetrar a la comunidad y empezar a discursar con la gente, con las mamás, con los papás, con los niños en su casa y ver la realidad de donde se genera la enfermedad: fue trascendente. Desde entonces a mí la Salud Pública me impactó[...]y no era la única persona; porque salíamos a campo y lo disfrutábamos. - iPor dios;, porque en muchos momentos no teníamos el vehículo para que nos transportaran, y no teníamos el dinero, nos íbamos caminando en ocasiones y no estaba cerquita. Porque era después de Xalisco [...] nunca dejamos de asistir a campo, nunca hubo una inasistencia y cuando sucede esa transformación de la escuela fue otro gallo, fue otra realidad. Creo que esa riqueza que tenía la escuela de medicina, fue crítico ese instante, se perdió, nunca fue rescatada. Fuimos privilegiadas las dos primeras generaciones y un poquito de la tercera; es el privilegio de contar con una metodología completamente diferente para interpretar a la realidad [...]". EE66
\end{abstract}

\title{
El conflicto universitario destruye el proyecto de Nueva Universidad
}

La segunda parte de la década del sesenta para la mayoría de las instituciones de educación superior de nuestro país, representó la travesía de un largo período de crisis política, académica y financiera, que atentó incluso contra su existencia misma. Fue una etapa donde abundaron; las demandas saláriales, la destitución de rectores, directores y profesores, así como en algunos casos su reincorporación, las firmas de contratos colectivos, el reconocimiento a sindicatos independientes, pagos de salarios atrasados, aumentos de becas y mejores condiciones para estudiar, revisiones de 
planes de estudios, problemas estudiantiles, apoyos a docentes y alumnos de otras instituciones; destrucción del patrimonio universitario, el asesinato de líderes estudiantiles, el encarcelamiento de profesores y alumnos. Así como la presencia de grupos de porros en los campi universitarios y su involucramiento en actos delictivos y de grupos policíacos.

Los avisos de una real confrontación con el gobierno del estado, iniciaron desde la campaña realizada por el coronel Rogelio Flores Curiel, candidato del PRI, quien viene antecedido de un desprestigio político, por su participación en los acontecimientos del jueves 10 de junio de 1971 (jueves de corpus) en la ciudad de México, siendo jefe de la policía; al tomar el cargo la lucha contra la universidad fue más frontal,

\footnotetext{
"Cuando Flores Curiel fue candidato, me llamó, para que le hiciera un plan para desaparecer la universidad, yo le decía:[...]Rogelio, cómo es posible que ya como gobernador no defiendas lo que significa la Universidad de Nayarit, si tú la quitas vas a desquiciar el patrimonio de cuando menos diez mil familias, vas a propiciar que todos los jóvenes se vayan del estado, la huida de cerebros; la universidad nunca se ha metido en cuestiones electorales, vas a quitar un centro de estudios a donde viene gente de varias partes a dar conferencias.
}

- jme importa poco; A mí me la quitas y si no dime cuando sales.

-Un día antes de que tú entres.

-Y así fue, tuve que dejar.

[...] inmediatamente reuní a todos mis colaboradores, directores y les informé de la situación diciéndoles que:

-La única manera en que no puede desaparecer la universidad es haciéndola autónoma.

Me fui con Gómez Reyes (gobernador), le informé lo que el Coronel quería, afortunadamente Gómez Reyes me mereció todo mi cariño, porque nunca, nunca me dio una consigna, nunca me dio una orden, nunca me comento, el me decía:

“-tu eres mi rector y la política universitaria tú la llevas, yo no me meto en eso, yo confío en ti- “.

- “Acá en la universidad, se está gestando la autonomía, usted ya va a salir, -vamos lográndola”- ;Adelante; , pero eso tienes que presentarlo ante el Congreso del Estado.

Fui al Congreso porque muchos diputados se oponían, la aprobaron [...]fueron logros de mi administración” (Entrevista, Ricardo Vidal Manzo, 1998)

Cuando toma posesión Rogelio Flores Curiel como gobernador del estado (1975-1981), la universidad ha logrado ya su autonomía, que fue una conquista de la comunidad universitaria, “[...]Los estudiantes y el personal académico de las escuelas superiores de la universidad participamos en el movimiento de autonomía y la obtuvimos[...] (Entrevista, Manuel Ulloa Herrero, 1998). 
Lograda la aprobación -por el congreso del estado-, se aprueba también la facultad de darse el estatuto $^{17}$ que debía regirla, primer documento en su tipo dentro de las instituciones de educación superior; el cual significaba que la universidad se daba así,

"la facultad para la renovación de sus estructuras académicas y administrativas en función de sus objetivos académicos. Si se requería otra estructura y se requería de la autonomía; con la autonomía la facultad de darse un estatuto y formularse un proyecto que fue discutido al que concurrieron y contribuyeron las diferentes escuelas con sus aportaciones, y finalmente se llevó al ámbito del Consejo Universitario para su aprobación” (Entrevista, Manuel Ulloa Herrero, 1998).

Con lo cual la universidad entra en un largo proceso de transformación académica y administrativa, en gran medida debido a la presencia del (IIE) ${ }^{18}$, el cual ayudaría a la universidad en las labores de planeación y evaluación académicas. Estas acciones se sistematizarían al apoyar el desarrollo académico de las diversas escuelas, la formación de investigadores y ayudar a formular el fideicomiso de las Unidades de Producción ${ }^{19}$.

El 16 de enero de 1977 en la torre de rectoría, se realizó un simposium donde se anunciaron reformas académicas y nuevos planes de estudio, bajo el propósito de desarrollar una enseñanza integral, para formar un universitario "interdisciplinario o multifacético". En esa ocasión se anunció la puesta en marcha del Plan Modular de la Escuela de Medicina Veterinaria, el Plan Experimental de Estudios para la Escuela de Economía, la Preparatoria Abierta, el PMGI A-36 para la Escuela de Medicina Humana "se pretende llevar al estudiante a aplicar una medicina comunitaria y formar en el educando una ideología de servicio social, para lo cual se estudiarán también las ciencias sociales[...]”, el Plan de Estudios de la Escuela de Agricultura, el Instituto de Ingeniería y las Unidades de producción “como medida fundamental para ligar el aspecto académico con el práctico, se habló de esfuerzos por crear tecnología intermedia, bajo una ideología nacionalista, con el fin de dar pasos serios hacia la eliminación de esa vieja universidad, que aún no puede borrar la formación de profesionistas con sentido elitista, de servicio y de defensa del sistema capitalista". De igual forma se habló sobre el aspecto de capacitación a los maestros universitarios, quienes en buena parte no cuentan con estudios a nivel de licenciatura; por ejemplo, los que hasta el momento se encuentran en el sistema de preparatorias; el $50 \%$ cuenta con estudios normalistas y solamente un $10 \%$ posee la licenciatura ${ }^{20}$.

\footnotetext{
${ }^{17}$ El Estatuto Jurídico, entra en vigor el 25 de enero de 1976, y sustituye a la ley orgánica. En su momento se consideró una de las leyes universitarias más avanzadas, con lo que la universidad puede atribuirse el derecho de autolegislar en materia de educación superior, mediante un consejo paritario de alumnos, maestros y trabajadores, su carácter democrático se manifiesta en la amplia participación para la designación de las autoridades, mediante elección directa y éstas sólo tienen carácter ejecutivo de las decisiones tomadas por el Consejo General Universitario.

${ }^{18}$ Creado a partir de un financiamiento tripartito UAN-CONACYT-Coordinación de Educación Superior SEP.

${ }^{19}$ El proyecto de las Unidades de Producción se presentó en la reunión de la ANUIES, celebrada en la ciudad de Veracruz, resultando ser uno de los proyectos aprobados. En esa ocasión le fue otorgado un fideicomiso de tres millones de pesos.

20 "Novedosos planes para formar la universidad" Periódico El Diario del Pacifico. 15 de enero de 1977.
} 
Éste proceso de innovaciones académicas y administrativas universitarias, observó una dinámica circular, donde se reorientan el proceso de enseñanza-aprendizaje, dando un viraje hacia un modelo; docencia-investigación-producción, propugnar por el establecimiento de una tecnología propia, allegarse recursos económicos para los centros, impulsar y readecuar carreras, contenidos curriculares, de acuerdo a las necesidades regionales y comunitarias.

El proceso inicia en las diversas escuelas, cuenta con la participación de los diversos institutos de investigación, complementada con las actividades en las unidades de producción y la capacitación de los trabajadores:

\begin{abstract}
"Se tienen funcionando en la actualidad: la granja avícola de Compostela, coordinada eficientemente por la Escuela de Medicina Veterinaria y Zootecnia; los barcos-escuela, la almadraba[...]para la captura de pesca de distintas variedades, la fábrica de harina de pescado y la unidad móvil-refrigerante para la venta de pescado al público[...] dentro de diez días[...]iniciará sus actividades la curtiduría de pieles (tenería mecanizada), dependiente de la Escuela Superior de Ingeniería Química Industrial[...]donde se procesarán diariamente más de cien pieles[...] ]en el mes de septiembre funcionará en Jala, la Casa de las Artesanías, con sus cinco áreas de producción: Madera, Hierro Forjado, Cerámica, Piel e Hilados y Tejidos. Se aprovecharán en esta Unidad de Producción, las experiencias de los artesanos de esa región y se convocará para que se integren a la misma [...] los estudiantes universitarios que tengan cualidades en algún área artesanal" ${ }^{21}$.
\end{abstract}

Por estas acciones, la Universidad Autónoma de Nayarit, puede, recibir subsidios extraordinarios a nivel federal:

"[...] ]en una reunión salimos cargados de pesos...se le explicó con mucha vehemencia al señor presidente Echeverría de que la universidad estaba a punto de morir. Se le presentó la panorámica de la universidad y se le expusieron las necesidades, nos dio un subsidio especial para pagar el pasivo, cinco millones de pesos, un subsidio especial de veintidós millones de pesos y para las Unidades de Producción en diciembre veinte millones más, para el Instituto de Investigaciones Jurídicas un subsidio especial mensual de \$250,000; siguió el Instituto de Pesquera, un cuarto de millón de pesos[...] (Rubén Hernández de la Torre, 1998).

Además la universidad está en posibilidades de establecer una diversidad de convenios interinstitucionales; con la UNAM se firman dos convenios para la descentralización académica, por su parte, la ANUIES y el CONACYT concedieron becas a estudiantes para estudiar en Australia, Argentina, Brasil y Japón. A la vez de recibir el reconocimiento de diversas instituciones:

\footnotetext{
“[...] fuimos invitados a México a una reunión Interamericana de Rectores de la América Latina, porque el presidente de la ANUIES era el licenciado Rangel Guerra, la UNAM fue el anfitrión, el IPN, UNL, [...] se discutieron bastantes temas relaciones con la universidad, entre ellos la autonomía y se puso como ejemplo la lograda por la Universidad de Nayarit. Había rectores de Argentina, Uruguay, Brasil, Chile, Venezuela, Perú, Cuba [...]un destacado investigador[...]al final hizo una pregunta a todo el presídium [...] se adelantó el licenciado Rangel Guerra.
}

21 “Las Unidades de Producción si funcionan” Periódico El Diario del Pacifico, 31 de julio de 1976. 
-[...] lo importante es la Universidad del año 2000, y esa universidad del año 2000 es la de Nayarit, que está vinculando el aprendizaje con la producción y el trabajo a través de sus Unidades de Producción.

El doctor Salomé se quedó sumamente entusiasmado y nos buscó [...] lse llevó un documento[...]para difundirlo en la América del Sur. Las Unidades de Producción le dieron proyección a la universidad, tuvimos visitas de funcionarios de alto nivel [...] ]el director del CONACYT, el rector de la Universidad Autónoma Metropolitana[...] (Entrevista, Rubén Hernández de la Torre, 1998).

Estas acciones fueron meritorias para que la universidad recibiera un nombramiento pocas veces visto, "[...] la UNESCO nombró a la Universidad de Nayarit, la "Universidad del año 2000", para que todas las universidades de América Latina fueran las que adoptaran ese proyecto; en la ANUIES está ese documento que para la universidad es histórico y debe recuperar" (Entrevista, Ricardo Vidal Manzo, 1998),

El turno de atropellos, robos, desalojos, agresiones, destrucción física y académica -como dice Federico Gómez Pombo (1977)- para la Universidad Autónoma de Nayarit, como el vivido por las universidades de Zacatecas, Guerrero, Puebla, Oaxaca, Guanajuato; llegó al segundo año del gobierno del coronel Rogelio Flores Curiel.

Para la (UAN), éste período aparte del saqueo material del cual fue víctima, la mayor agresión que pudo haber sufrido, se reflejó en el ataque frontal a sus programas académicos y proyectos de investigación. Una muestra de esto, -y que marca el inicio de la confrontación- fue el ataque perpetrado, contra las instalaciones del (IIE), ubicado en la población de Xalisco, la madrugada del 17 de marzo de $1977^{22}$, cuando sus instalaciones fueron atacadas por grupos de porros del "Movimiento Organizado Democrático Universitario" (MODU) y de la "Unidad Liberal Benito Juárez”, quienes a punta de zapapicos y disparos de arma de fuego a sus cerraduras, destruyeron parte de sus instalaciones y varios de sus enseres: máquinas de escribir, sillas, escritorios, fueron saqueados y llevados a la sala de maestros y baños de la escuela preparatoria número $1^{23}$ :

\begin{abstract}
"La madrugada fueron sacados los archivos con las investigaciones, los expedientes de los alumnos, de los planes experimentales, así como maquinaria de impresión, archivos, libros e inclusive un mapa con fotografía aérea de Nayarit que servía de base para un estudio de tenencia de la tierra[...] Jse agregó una curiosa ceremonia: pilas de libros fueron balaceados. Los temas de sociología y la economía recibieron una rociada de plomo. También -por supuesto- los de la ciencia política" (Revista Proceso, 21-26 1977).
\end{abstract}

Con estos actos vandálicos, se interrumpen las actividades académicas y de investigación

\footnotetext{
22 Periódico El Diario del Pacifico, 18 de marzo de 1977.

${ }^{23}$ El Diario del Pacifico durante su existencia, dio una cobertura amplia a los sucesos universitarios. De acuerdo a los registros el primer artículo universitario aparece el 4 de septiembre de 1975, con el artículo sobre la lucha por la autonomía en la UNI-NAY, finalizando el 10 de agosto de 1978 con el artículo en el cual se debate sobre la no admisión de embarazadas a la escuela de enfermería.
} 
universitarias. El proyecto de Nueva Universidad, que asentaba una gran parte de sus actividades en el programa de las Unidades de Producción, se ve seriamente agredido y en cuestión de semanas se destruye. Se nombra una Junta Provisional de Gobierno, quienes en una asamblea, por votación unánime logran su designación y con el respaldo de la SEP, destituyen al entonces rector Rubén Hernández de la Torre, a los vicerrectores, el secretario general, el abogado y otros funcionarios universitarios.

Este hecho tiene sus consecuencias hacia las actividades normales que tenían las facultades y escuelas, por ejemplo en la Facultad de Ciencias de la Educación, se recibe un oficio en el cual se señala que deben suspenderse todas las actividades en el plantel (académicas, técnicas y administrativas), debido a que no se ha... "encontrado el acta del H. Consejo General Universitario, sobre la aprobación del proyecto de funcionamiento de esa Facultad de Ciencias de la Educación y

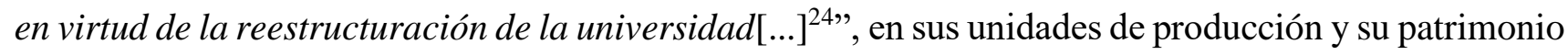
en equipo, infraestructura, material bibliográfico y transportes.

Ante éste hecho, una gran cantidad de profesores tanto nacionales como extranjeros que participaban de convenios interinstitucionales, en diversos proyectos universitarios dentro de los institutos de investigación, unidades de producción y realizando docencia en distintas escuelas, son despedidos, cuando no agredidos. De esta manera aquel programa académico que en su tiempo permitió que la UAN fuera considerada, la universidad del año 2000, termina en el mes de julio de 1977. Después la universidad entra en un largo año de huelga.

\section{El conflicto universitario hace crisis en la escuela de medicina}

El mes de junio de 1977 marca el final de la primera etapa del proyecto de la escuela de medicina, las autoridades (triunvirato) recién nombradas por el Consejo General Universitario, toman diversas decisiones; entre ellas la desaparición de la Facultad de Ciencias de la Educación, del Instituto de Ingeniería y del de Investigaciones Económicas. En el caso de los docentes, bajo el argumento de la tendencia comunista, y como una muestra de liberar a la institución de estas tendencias políticas, se empezaron a retener los sueldos y se intensificaron las presiones para despedir a muchos profesores, "Si, fue cuando la gran migración de aquellos docentes que iban de todas partes del país, del estado incluso y del extranjero[...]"..EE69

Una de las escuelas más afectadas fue la de medicina, porque experimenta la renuncia de la mayoría de sus tutores. Acto con el que se ven interrumpidas drásticamente sus actividades académicas,

\footnotetext{
${ }^{24}$ Periódico El Diario del Pacifico, 17 de junio de 1977.
} 
"[...]cuando la doctora Pura quería renunciar y..ya se estaba sintiendo la presión porque a la doctora Pura le comenzaron a llegar anónimos, le comenzaron a decir que se pusiera en paz, que organizara bien a sus grupos, a sus alumnos que no los mal informara[...]'EE77

\section{Se inician cambios sustanciales al programa de la escuela de medicina}

Para la escuela de medicina, el conflicto universitario tiene una repercusión fundamental hacia la estructura original de su programa de estudios, algunos de los cambios que se originan son por ejemplo; que los tutores originales son presionados a renunciar, dándose una renuncia masiva, hay una baja sensible en el ritmo normal de las actividades académicas; aún con instalaciones tomadas, las labores continúan en otros espacios, que son temporalmente prestados, las actividades en clínica siguen con su programación habitual, sin embargo el conflicto universitario llega a afectar el año lectivo:

\footnotetext{
"Se viene el conflicto con los tutores, se viene el conflicto en la universidad, tenemos prácticamente un año perdido, cuando estábamos en tercer año[...]tratamos de mantener las clases, casi no perdimos clases de manera extraoficial, pero de manera oficial estábamos en huelga, de manera oficial no hubo actividades, de manera oficial andaba desapareciendo la escuela de medicina, en tercer año" EE80
}

De esta manera, se ven afectadas las actividades en aula, en comunidad, las prácticas de laboratorios, y de una manera importante el ambiente escolar vivido por alumnos y docentes antes del período de crisis. El conflicto universitario, para el programa académico de la escuela de medicina, es el parteaguas de un proyecto académico en construcción durante un año y medio y los cambios que empieza a sufrir a partir del reinicio de las actividades escolares.

Todas las escuelas de la universidad sufrieron en distinto grado y severidad las consecuencias del conflicto universitario, dentro de las más afectadas puede ubicarse a la escuela de medicina:

\footnotetext{
"Teníamos mucha confusión, desasosiego, estábamos intranquilos, sabíamos de los ambientes universitarios, aquella "jodida" huelga que nos vino a trastocar y que a pesar de que nosotros asistíamos a la escuela, que nos tildaron de esquiroles y que se presentaron los exámenes de cada uno de los módulos, nos hacen repetir los módulos. Muchos de nuestros valiosos compañeros se tuvieron que retirar y emigran a otras universidades, porque aquí era un mazacote. Momentos de pena, de dolor, agresiones a la escuela de medicina, robos a la escuela de medicina. Nosotros haciendo guardias con todo el temor del mundo para que no penetraran a la escuela de medicina, fue caótico, caóticos esos momentos”.EE81
}

El reinicio de las actividades universitarias, representó un cambio drástico en la forma en que venía desarrollando sus labores. Inicia con una nueva dirección y subdirectores, se hace necesario cubrir las vacantes que dejaron los primeros tutores, se entra en un largo proceso de discusión para oficializar o no las actividades que se desarrollaron durante el período de huelga, se inician nuevas contrataciones de maestros que requerirán los alumnos de tercer año, entre otras cuestiones. 
Los cambios empiezan a percibirse en distintos órdenes, queremos rescatar lo que sucedió en algunas áreas que nos parecen importantes; ¿cuáles fueron los cambios que los alumnos percibieron del ambiente estudiantil?, ¿qué ocurrió con las actividades en comunidad?, ¿dentro del aula cuales fueron las novedades; en las actividades, horarios, relación docente-alumno?, ¿cómo continuaron las actividades en el área clínica?, ¿la filosofía del programa cómo se reorientó?:

"[...]por familiares que han estado en la escuela de medicina, yo creo que definitivamente no solo se perdió la filosofía del plan A-36, se perdieron muchas cosas, por ejemplo la visión del médico en cuanto a la problemática de su entorno en el aspecto de salud pública y en el aspecto de la situación económica de las clases más marginadas que tenemos y tal parece que eso ya no existe”.EE82

Con los procesos de reforma universitaria iniciados en el año de 1999 y su implementación en los inicios del dos mil. El PMGI A-36, llega a su fin y en su lugar aparece el nuevo programa por Competencias Profesionales Integradas. El cual en el año de 2008 recibió su primera acreditación y su re-acreditación en el 2013 por parte del COMAEM. 


\section{BIBLIOGRAFÍA CONSULTADA.}

Abreu Hernández Luis Felipe, et.al. "Programa de Calidad de la Educación Médica. Sistema Nacional de Acreditación", Asociación Mexicana de Facultades y Escuelas de Medicina, A. C. México 1995.

ANUIES. "Matricula y personal docente de la carrera de medicina en México 1984"

Atkinson Robert “The life story intereview” Qualitative Research Methods, vol. 44, Sage Publications. USA, 1998.

Barba Casillas Bonifacio (Coordinador) 2000 "Orígen y desarrollo de la Universidad Autónoma de Aguascalientes 1973-1998”, 1a. Ed. Universidad Autónoma de Aguascalientes.

Fernández Pérez Jorge A. "Las carreras de medicina en México” Revista Perfiles Educativos, No. 73, 1996.

García Colorado Carmen y Sara Morales López. "Experiencia innovadora en el campo de la formación del médico: El Plan A-36”. Perfiles Educativos. CISE-UNAM, Año 1993, no. 59

García Ferrarndo Manuel "La encuesta" en El análisis de la realidad social. Métodos y técnicas de investigación. Ed. Alianza. Madrid. 1986

González, Wood Hernán (1988) "Proyecto de Readecuación curricular del Programa de Medicina General Integral A-36”. UNAM

Historia de la Facultad de Medicina Universidad de Colima. (www.ucol.edu.mx $)$

Morales, Acosta Elvia, María Susana Nolasco González, Andrea Cibrián Pérez, Bernabé Ríos Nava, (1999). La Conciencia Histórica de la Universidad Autónoma de Nayarit. $1^{\text {a }}$. ed. Dirección de Innovación Educativa. Tepic, Nayarit. mexico.

Padua Jorge e Ingvar Ahmna, (2002) La organización de un "survey” en Técnicas de Investigación Aplicadas a las Ciencias Sociales. El Colegio de México-Fondo de Cultura Económica. 9ª Reimp. México 2002.

Proyecto para la creación de la Escuela de Medicina de la Universidad Autónoma de Nayarit. Fotocopia.

Salinas, Amescua Bertha, (1977). Una alternativa de la universidad tradicional. La experiencia de la Universidad Autónoma de Nayarit (México). Revista del Centro de Estudios Educativos. VII, (2)

Sandoval, Lara Zeferino, (1975). La universidad no será escentario de batalla política: Vidal Manzo. Periódico Diario del Pacífico.

Soberón Guillermo y José Manuel Álvarez Manilla "El marco internacional de la educación médica”, Gaceta Médica de México, vol. 127, No. 2 México 1991. 
Taylor S.J. \& R. Bogdan “Introducción a los métodos cualitativos de investigación” $3^{\mathrm{a}}$. Reimp. Ed. Paidós Básica. Barcelona. Buenos Aires. México. 1996

Universidad Autónoma de Nayarit (1974). Acta de la sesión celebrado por el H. Consejo General Universitario, el lunes 8 de julio de 1974. Fotocopia

UNAM (1978) “Plan de Estudios 1978”, 3ª ed. Dirección General de Publicaciones.

UNAM (1988) "Proyecto de Readecuación Curricular del Programa de Medicina General Integral" Vega Prado Rodolfo, et. al. (1990) "Actitud del alumno del Programa de Medicina General Integral en el trabajo de comunidad”. Revista Mexicana de Educación Médica, 1 (3)

Vela Peón Fortino "Un acto metodológico básico de la investigación social: la entrevista cualitativa". En "Observar, escuchar y comprender sobre la tradición cualitativa en la investigación social”.Tarre, Ma. Luisa (Coordinadora), 1ª. Ed. Miguel Ángel Porrúa-El Colegio de MéxicoFLACSO, México 2001

"Novedosos planes para formar la universidad" Periódico El Diario del Pacifico. 15 de enero de 1977. "Las Unidades de Producción si funcionan” Periódico El Diario del Pacifico, 31 de julio de 1976.

Zamudio, Vidal Carlos, (1974). Revolucionaria innovación llevará a cabo el rector Vidal Manzo. Se crearán bajo el patrocinio de la universidad varias pequeñas industrias. Periódico Diario del Pacífico. 